\title{
Turn-by-Turn Analysis of Proton and Gold Beams at Injection in the AGS Booster*
}

\author{
C. Gardner, L. Ahrens, N. Williams, BNL, Upton, NY 11973, USA \\ Abstract

\section{DATA COLLECTION}

In this paper we describe the latest version of a program we have used for several years to acquire and analyze turn-byturn data from pick-up electrodes in the AGS Booster during injection. The program determines several parameters of the injected beam including the tunes and the position and angle of the incoming beam. Examples are given for both proton and gold injection.

\section{INTRODUCTION}

Several years ago we developed a computer program called "PIP" [1] to acquire and analyze turn-by-turn data from PUEs (Pick-Up Electrodes) in the AGS during injection. Originally, the turn-by-turn data were generated by a halfturn pulse of beam (some $2.4 \mu$ s long) that was injected and ailowed to pass turn-by-turn through a given PUE. The resulting signals were digitized and the digital data were processed to yield the position of the beam on each pass through the PUE. A several-parameter function was then fitted to the position-versus-turn data yielding, among other things, the tune and the position and angle of the injected beam. Recently we developed another method for generating the turn-by-turn data in which a half-turn gap or "hole" (typically 1 to $10 \mu$ s wide) is chopped out of a long beam pulse before the pulse is injected into the synchrotron. When the "hole" enters the machine and passes turn-by-turn through a PUE, it produces a signal similar to that of a single half-turn of beam. The advantage of this technique is that it neither interrupts the machine-physics processes occurring during the injection stacking nor (on a more practical level) does it interrupt the normal delivery of beam to the Physics programs. This has been particularly useful for studying the injection of gold and other heavy ions in the AGS Booster [2].

The program originally employed stand-alone digitizers. These were eventually replaced with multi-channel digital oscilliscopes which perform the necessary digitization and storage of data and at the same time provide an instantaneous picture of the signals to be analyzed; this makes setup and verification of the signals very easy. The program is now also ablc to analyze the coupling between the horizontal and vertical planes. This is essential for detailed studies of heavy ion injection in the Booster where coupling is used to enhance the injection efficiency [2]. Following is a detailed description of the most recent version of the program, now used primarily to study and monitor the injection of protons and heavy ions in the Booster.

\footnotetext{
* Work supported by the U.S. Department of Fnergy
}

Before digitization, the signals from the plates of a given PUE assembly are combined to produce a SUM signal proportional to the total charge inside the PUE and a DIFFerence signal proportional to the horizontal or vertical displacement of the beam in the PUE. The SUM and DIFF signals are piped from the Booster ring to the MCR (Main Control Room) where they are connected to a multichannel digital oscilliscope. We are currently using LeCroy 9304A (Quad $200 \mathrm{MHz}$ ) or 9414 (Quad $150 \mathrm{MHz}$ ) oscilliscopes for this purpose. The digitized data are transfered from the oscilliscopes to Sun work stations in the MCR via a National Instruments GPIB-ENET IEEE 488 controller. A LabView program collects the data. Figures 1 and 2 show typical digitized SUM and DIFF signals generated by a half-turn of protons injected into the Booster and observed for some 40 turns. (The revolution period here is $1.2 \mu \mathrm{s}$.)

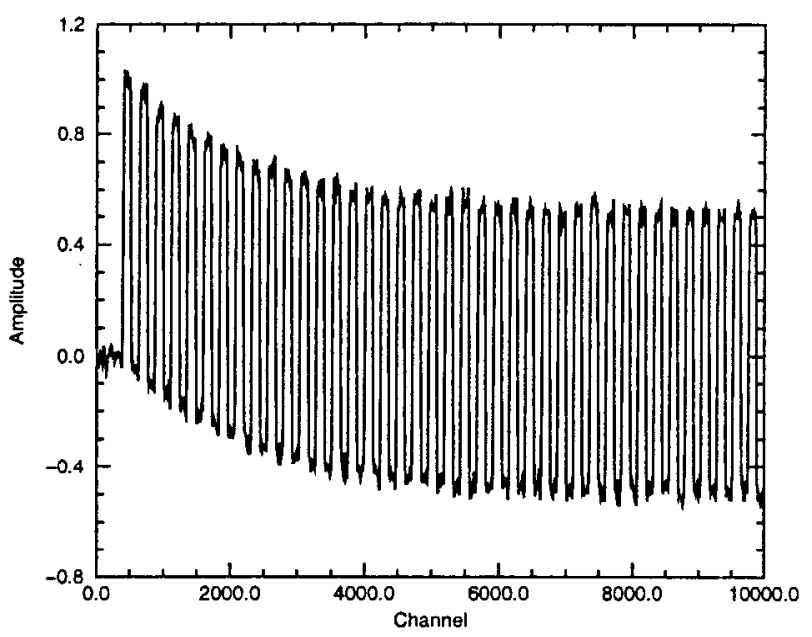

Figure 1: Digitized PUE SUM Signal.

\section{DATA ANALYSIS}

The digitized SUM and DIFF signals collected from the oscilliscope are analyzed by a collection of Fortran subprograms. The analysis begins with the SUM data which serve as a set of markers for the times when beam enters and exits the PUE. Specifically, the well-defined "edges" in the SUM signal allow one to determine precisely when beam enters and exits the PUE on each turn around the machine. This eliminates the need for any synchronization of the digitization with the revolution period. Moreover, it allows one to establish local baselines for determining the signal amplitude for each pass through the PUE. For each turn then, 


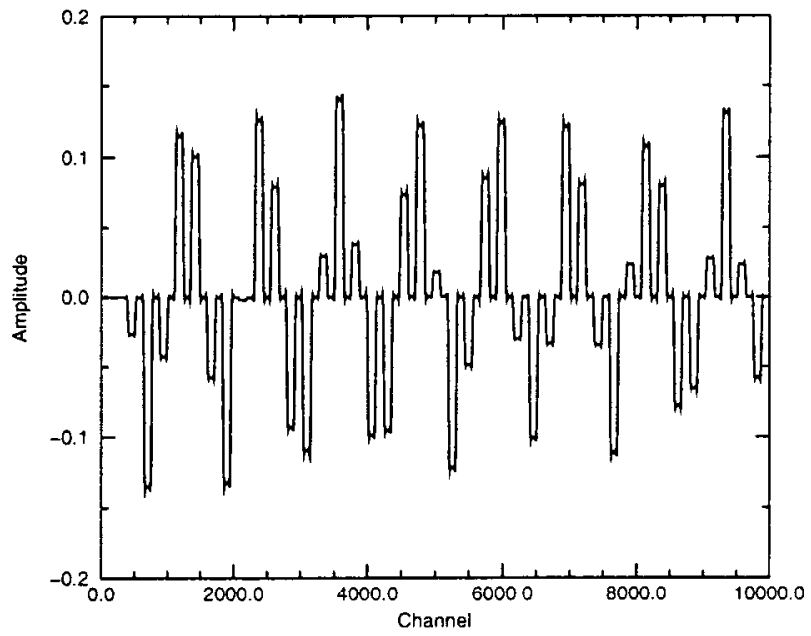

Figure 2: Digitized PUE DIFF Signal.

the digitization sample numbers (labeled "Channel" in Figures 1 and 2) corresponding to the interval of time when beam is inside the PUE are located and the average value of the SUM signal (with respect to the local baseline) is computed over those sample numbers. The value of the DIFF signal (with respect to its local baseline) is averaged over the same sample numbers for each turn. In this way one obtains an average SUM and an average DIFF value for each turn. The position, $X_{n}$, of the beam in the PUE on the $n$th turn is then just a constant (which depends only on the geometry of the PUE) times the DIFF value divided by the SUM value for that turn. We have found this treatment of the SUM and DIFF data to be particularly robust; even DIFF signals with rather poor signal-to-noise ratios yield surprisingly good position-versus-turn data.

$\Lambda$ Fast Fourier Transform [3] of the position-versus-turn data gives the tune and initial phase of the betatron oscillations at the PUE. Using these as starting values, a function of several parameters is fitted [4] to the data. For the case in which there is no coupling between the horizontal and vertical planes, the fitting function is [1]

$$
X_{n}=C+D n+A e^{-f(n)} \cos \psi(n)
$$

where

$$
f(n)=\{n \pi \Delta Q\}^{2} / 2
$$

and

$$
\psi(n)=2 \pi n\left\{Q_{0}+n \delta Q / 2\right\}+\phi .
$$

Here the seven parameters, $C, D, A, Q_{0}, \phi, \Delta Q$, and $\delta Q$ are, respectively, the position of the closed orbit at the PUE; the change in the closed orbit position per turn; the amplitude, tune and phase of the betatron oscillations; the tunespread of the beam; and the tune-shift per turn. Figure 3 shows the position-versus-turn data and fitted curve obtained from the SUM and DIFF signals of Figures 1 and 2. The corresponding FFT is shown in Figure 4. The values of the fitted parameters are given in Table 1.

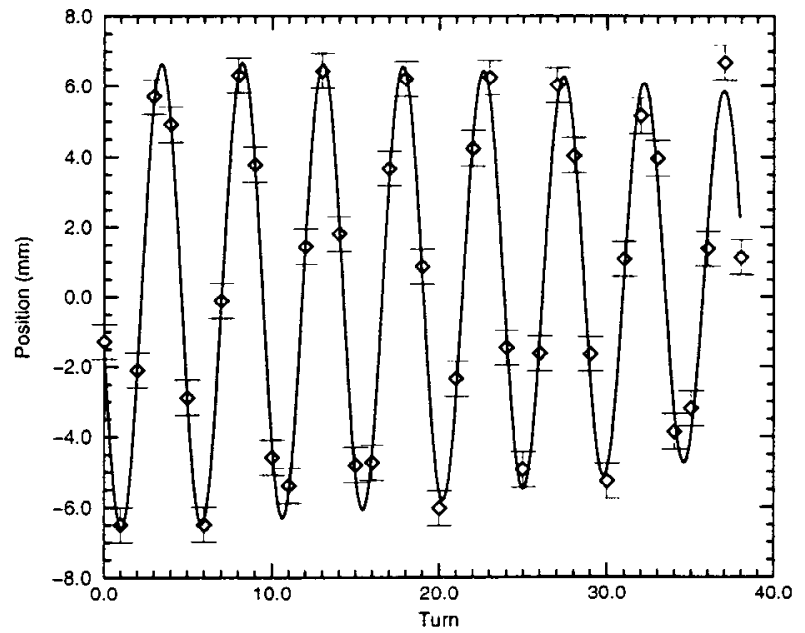

Figure 3: Position-vs-Turn Data and Fitted Curve.

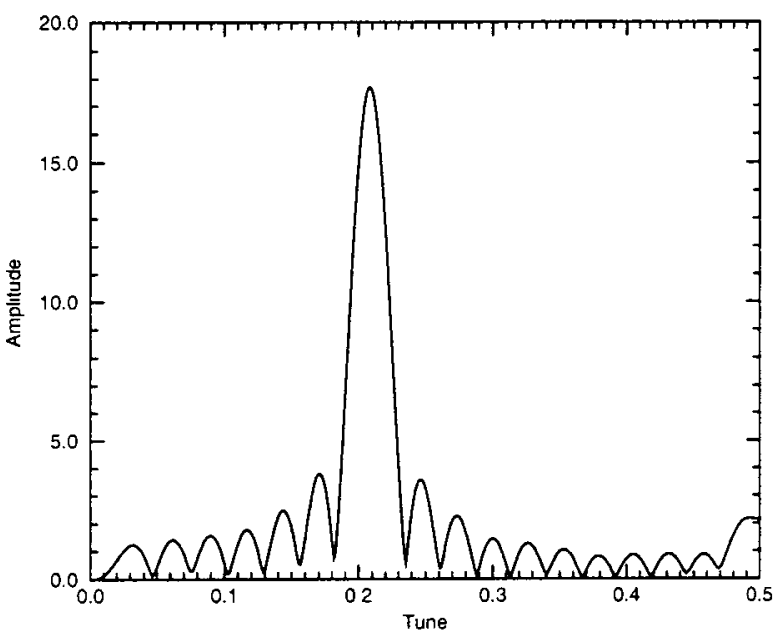

Figure 4: FFT of Position-vs-Turn Data.

The initial position and angle, $X_{p}$ and $X_{p}^{\prime}$, of the beam at the PUE are given by

$$
\begin{gathered}
X_{p}=A \cos \phi \\
X_{p}^{\prime}=-(A / \beta)\{\alpha \cos \phi+\sin \phi\}
\end{gathered}
$$

where $A$ and $\phi$ are the fitted amplitude and phase, and $\alpha$ and $\beta$ are the Courant-Snyder lattice parameters at the

Table 1: Fitted Parameters (Uncoupled Case)

\begin{tabular}{|c|c|c|}
\hline$C$ & $0.0(2)$ & $\mathrm{mm}$ \\
\hline$D$ & $0.02(1)$ & $\mathrm{mm} /$ turn \\
\hline$A$ & $6.6(2)$ & $\mathrm{mm}$ \\
\hline$Q_{0}$ & $4.792(1)$ & tune \\
\hline$\phi$ & $0.283(8) \times 2 \pi$ & radians \\
\hline$\Delta Q$ & $0.006(1)$ & tune-spread \\
\hline$\delta Q$ & $-0.00002(5)$ & tune/turn \\
\hline
\end{tabular}


PUE. The transfer matrix from the PUE to the point of injection then gives the initial position and angle of the beam at injection. The lattice parameters and transfer matrix are obtained from the MAD [5] code.

For the case in which there is coupling between the planes, the fitting function is taken to be

$$
X_{n}=C+D n+A_{1} \cos \psi_{1}(n)+A_{2} \cos \psi_{2}(n)
$$

where

$$
\psi_{1}(n)=2 \pi n Q_{1}+\phi_{1}, \quad \psi_{2}(n)=2 \pi n Q_{2}+\phi_{2} .
$$

Here we assume that the tune-shift per turn and the loss of coherence due to tune-spread are negligible. The six parameters $A_{1}, A_{2}, Q_{1}, Q_{2}, \phi_{1}, \phi_{2}$ are the amplitudes, normal-mode tunes, and initial phases of the coupled betatron oscillations. Figure 5 shows the position-versus-turn data and fitted curve obtained for gold ions in the Booster with linear coupling introduced during injection. The corresponding FFT in Figure 6 clearly shows the two normalmode tunes. The values of the fitted parameters are given in Table 2. As with the uncoupled case, the program calcu-

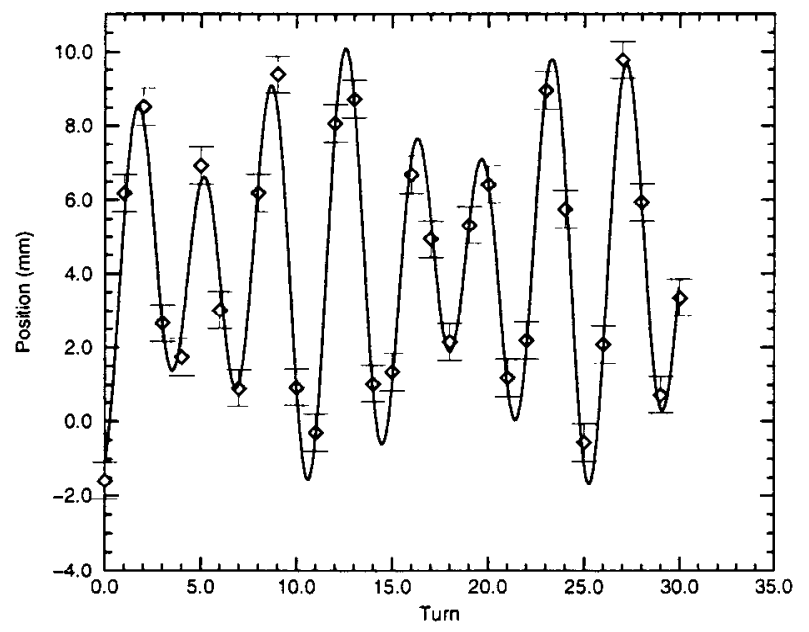

Figure 5: Position-vs-Turn Data and Fitted Curve.

Table 2: Fitted Parameters (with Coupling)

\begin{tabular}{|c|c|c|}
\hline$A_{1}$ & $4.2(1)$ & $\mathrm{mm}$ \\
\hline$A_{2}$ & $1.8(1)$ & $\mathrm{mm}$ \\
\hline$Q_{1}$ & $4.726(1)$ & tune \\
\hline$Q_{2}$ & $4.800(1)$ & tune \\
\hline$\phi_{1}$ & $0.58(1) \times 2 \pi$ & radians \\
\hline$\phi_{2}$ & $0.44(2) \times 2 \pi$ & radians \\
\hline
\end{tabular}

lates the initial position and angle of the beam at the PUE using the fitted parameters and the lattice parameters at the PUE. The explicit formulae used are given in Ref. [6]. The transfer matrix from the PUE to the point of injection then gives the initial position and angle of the beam at injection.

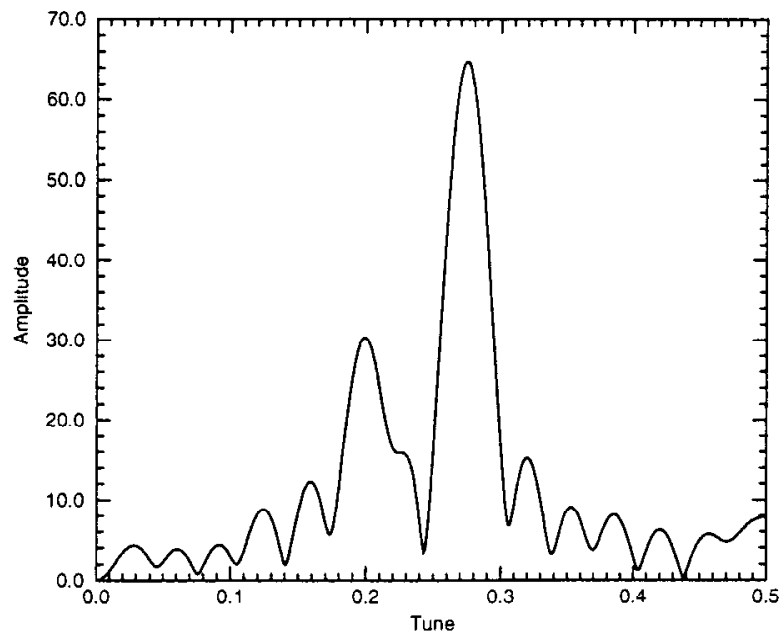

Figure 6: FFT of Position-vs-Turn Data.

\section{REFERENCES}

[1] C. Gardner and L. Ahrens, IEEE Transactions on Nuclear Science, Vol. NS-32, No. 5, October 1985, pp. 1888-1890.

[2] C. J. Gardner, "Injection of Gold Ions in the AGS Booster with Linear Coupling", Proceedings of the 1999 Particle Accelerator Conference.

[3] W. H. Press, et. al., Numerical Recipes in Fortran, 2nd edition, Cambridge University Press, (1992). pp. 498-508

[4] W. H. Press, et. al., pp. 678-682

[5] H. Grote and F. C. Iselin, "The MAD Program Version 8.4 User's Reference Manual", CERN/SL/90-13 (AP), $27 \mathrm{Au}$ gust 1991.

[6] C. J. Gardner, "Formulae for the PIP Analysis of Linear Coupling", AGS/AD/Tech. Note No. 476, March 30, 1998. 